\title{
SOME OPERATIONS PRESERVING LOG-CONCAVITY OF NONNEGATIVE FUNCTIONS
}

\author{
VADIM E. LEVIT AND EUGEN MANDRESCU
}

Abstract. In this paper we introduce the notions of $k$-log-concave and $k,[p, q]$-concave functions, as generalizations of log-concave and concave sequences, respectively. We also present sufficient conditions under which some operations with such functions yield $k$-log-concave functions. In particular, we infer that the convolution of a positive 1 -log-concave sequence and a nonnegative sequence of $k+1$ elements is $k$-log-concave.

Mathematics subject classification (2000): 26A51, 05A20, 26 D99.

Key words and phrases: log-concave, $k$-log-concave, concave, $k,[p, q]$-concave.

\section{REFERENCES}

[1] E. A. BENDER, E. R. CANFIELD, Log-concavity and related properties of the cycle index polynomials, Journal of Combinatorial Theory A, 64, (1996), 57-70.

[2] A. Berenstein, A. VAinshtein, Concavity of weighted arithmetic means with applications, Archives of Mathematics (Basel), 69, (1997), 120-126.

[3] F. BRENTI, Log-concave and unimodal sequences in algebra, combinatorics and geometry: an update, Jerusalem Combinatorics '93, Contemporary Mathematics, 178, (1994), 71-89.

[4] G. D. GodsiL, Algebraic combinatorics, Chapman \& Hall, New York, 1993.

[5] S. KARLIN, Total positivity, Stanford University Press, Stanford, CA, 1968.

[6] J. KeILson, H. Gerber, Some results for discrete unimodality, Journal of the American Statistical Association, 334, (1971), 386-389.

[7] R. P. STANLEY, Log-concave and unimodal sequences in algebra, combinatorics, and geometry, Annals of the New York Academy of Sciences, 576, (1989), 500-535.

[8] Y. WANG, Linear transformations preserving log-concavity, Linear Algebra and its Applications, 359, (2003), 161-167. 